\title{
Evidências de Validade e Normas do TEPIC-M para crianças e adolescentes
}

\author{
Luana Metta, Rodrigo da Silva Maia, Daniele Caroline Leôncio \\ Laura Aragão, Hedyanne Guerra Pereira, Eulália Maria Chaves Maia, Izabel Hazin ${ }^{1}$ \\ Universidade Federal do Rio Grande do Norte, Natal-RN, Brasil
}

\section{RESUMO}

O TEPIC-M é um teste que avalia a memória visual de curto prazo, validado e normatizado para a faixa etária de 17 a 89 anos, com o objetivo de encontrar evidências de validade de critério e normas para crianças e adolescentes. Este estudo contou com 542 estudantes com idade entre seis e 16 anos, de instituições de ensino públicas e privadas da cidade de Natal/RN. Verificou-se diferença estatisticamente significativa na média de acertos quando comparados os desempenhos dos participantes em função do tipo de escola $(p<0,001)$, bem como em função da idade $(p<0,001)$. Por outro lado, não foram verificadas diferenças na média de acertos entre sexos $(p=0,808)$. Conclui-se que o instrumento é adequado para a avaliação da memória visual de curto prazo entre crianças e adolescentes brasileiros, podendo ser utilizado em futuros estudos com essa população.

Palavras-chave: memória de curto prazo; avaliação psicológica; psicometria.

\section{ABSTRACT - Evidences of validity and standards of the TEPIC-M for children and adolescents}

The TEPIC-M is a test that evaluates short-term visual memory that has been validated and standardized for the 17 to 89 years age group. Participants of this study were 542 students aged between six and 16 years, of public and private educational institutions in the city of Natal, Brazil. The study aimed to find evidence of criterion validity and standards for children and adolescents. Statistically significant difference were found in the mean number of correct answers when comparing the performances of the participants according to the type of school $(p<0.001)$ and age $(p<0.001)$. Conversely, there were no differences in the mean number of correct answers between the sexes $(p=0.808)$. It was concluded that the instrument is suitable for the evaluation of short-term visual memory among Brazilian children and adolescents and can be used in future studies with this population.

Keywords: short term memory; psychological assessment; psychometrics.

\section{RESUMEN - Evidencias de validez y normas de la TEPIC-M para niños y adolescentes}

El TEPIC-M es un test que evalúa la memoria visual a corto plazo, validada y regulada para la franja etaria de 17 a 89 años. Con el objetivo de encontrar evidencias de validez de criterio y normas para niños y adolescentes, este estudio contó con 542 estudiantes con edades entre seis y 16 años de instituciones de enseñanza pública y privada de la ciudad de Natal - RN. Se verificó diferencia estadísticamente significativa en la media de aciertos cuando se compararon los resultados de los participantes en función del tipo de escuela $(p<0,001)$, así como en función de la edad $(p<0,001)$. Por otro lado, no se verificaron diferencias en la media de aciertos entre sexos $(p=0,808)$. Se concluye que el instrumento es adecuado para la evaluación de la memoria visual de corto plazo entre niños y adolescentes brasileños, pudiendo ser utilizado en futuros estudios con esa población.

Palabras clave: memoria a corto plazo; evaluación psicológica; psicometría.

Ao longo do desenvolvimento das ciências cognitivas, percebe-se a proposição de diferentes modelos explicativos e classificatórios para a memória, dentre os quais se destaca o modelo das memórias de curto e longo prazo, com diferenças significativas em termos da capacidade de armazenamento (limitada ou ilimitada) e duração (segundos em contraste com décadas). Tal distinção é central para o entendimento dos dois principais modelos mnêmicos, a saber, modelo de multiarmazenamento e modelo de armazenamento unitário (Eysenck, 2017).

Atkinson e Shiffrin (1968) propuseram modelo de multiarmazenamento, no qual a arquitetura básica da memória seria explicada por processamentos sequenciais. Inicialmente, os estímulos ambientais seriam processados e armazenados de forma específica para cada modalidade sensorial, com duração extremamente breve.

${ }^{1}$ Endereço para correspondência: CCHLA - Departamento de Psicologia, Campus da UFRN - Lagoa Nova, Avenida Senador Salgado Filho, s/n, 59078-000, Natal, RN. E-mail: izabel.hazin@gmail.com

Agradecimentos ao Conselho Nacional de Desenvolvimento Científico e Tecnológico (CNPq) e à Coordenação de Aperfeiçoamento de Pessoal de Nível Superior (CAPES) pelas bolsas de estudos e produtividade que viabilizaram a realização deste estudo. 
Caso houvesse investimento atencional sobre o estímulo, este passaria para a memória de curto prazo, cuja capacidade seria limitada. Por fim, essa informação poderia finalmente ser transferida para a memória de longo prazo, mais duradoura e ilimitada. Porém, estudos criticaram tal modelo e evidenciaram que o armazenamento pode ocorrer de forma não linear, ou seja, em paralelo entre sistemas de memórias diferentes (Abreu, Lima, Siquara, Wyzykowski, \& Fonseca, 2018).

Posteriormente, foi sugerida uma substituição do modelo multiarmazenamento por um modelo de armazenamento unitário, no qual a memória de curto prazo poderia ser compreendida enquanto uma ativação temporária de representações que se encontravam na memória de longo prazo ou de representações de elementos que foram percebidos recentemente (Jonides et al., 2008). No entanto, esse modelo não explicava os diferentes tipos de ativação da memória de longo prazo, assim como não corroborava os achados clínicos de pacientes amnésicos que apontavam o papel preponderante do hipocampo na memória de longo prazo em comparação com a memória de curto prazo (Eysenck, 2017).

No que se refere, de modo específico, à memória de curto prazo, uma abordagem propôs a substituição do modelo unitário por um sistema de múltiplos componentes, que tem como objetivo primordial facilitar, por meio do armazenamento e da manipulação, a execução de atividades cognitivas complexas, tais como aprendizagem e raciocínio. Esse modelo, denominado memória de trabalho, é composto por quatro componentes armazenadores de curto prazo: o executivo central, a alça fonológica, o esboço visuoespacial e, mais recentemente, o buffer episódico (Baddeley, 2012; Baddeley \& Hitch, 1974; Rueda \& Sisto, 2007).

O componente executivo central possui capacidade limitada, assemelha-se à atenção e trabalha com quaisquer tipos de tarefa com diferentes demandas cognitivas. A alça fonológica e o esboço visuoespacial são considerados sistemas escravos, mobilizados pelo executivo central para fins específicos. A alça fonológica proporciona o armazenamento da ordem em que as palavras são apresentadas, enquanto o esboço visuoespacial é utilizado para armazenamento e manipulação de padrões visuais e de movimento espacial. Por fim, o buffer episódico tem como papel articular a memória de trabalho com a percepção e com a memória de longo prazo, bem como integrar informações visuais e auditivas (Baddeley, 2000; Eysenck, 2017).

Porém, destaca-se que, apesar da memória de curto prazo ter sido funcionalmente assimilada à memória de trabalho, esta parece depender de estruturas cerebrais distintas (Abreu et al., 2018). A dimensão visual da memória de curto prazo desempenha papel crucial em atividades cotidianas, como reconhecimento de objetos, construção de imagens mentais, orientação espacial, aprendizagem e raciocínio visoespaciais
(Baddeley, 2012; Garcia, Mammarella, Pancera, Galera, \& Cornoldi, 2015).

Do ponto de vista do desenvolvimento, pesquisas apontam que esse componente se desenvolve ao longo da infância e da adolescência. As habilidades para estabelecer associações entre as informações visuais e a capacidade de memória parecem variar de acordo com a idade. É interessante notar que crianças e idosos têm maior dificuldade para manter na memória a localização espacial de objetos quando comparados a adultos jovens. Essa forma de $\mathrm{U}$ invertido (retrogênese), na qual se identifica um paralelo entre aquisição e declínio de habilidades específicas, parece estar vinculada às habilidades da memória na infância e velhice (Brockmole \& Logie, 2013; Gómez et al., 2018; Roberts, Strait, \& Decker, 2018; Swanson, 2017).

No entanto, apesar da importância e da independência da dimensão espacial da memória de curto prazo em relação ao domínio verbal, notadamente em termos da aquisição e desenvolvimento de habilidades acadêmicas matemáticas e de leitura e escrita (Montoya et al., 2019), ela não tem obtido o mesmo interesse que esta última, seja em termos de pesquisas realizadas ou da proposição de instrumentos que a avaliem. Em consulta à lista do Sistema de Avaliação de Testes Psicológicos (SATEPSI), identificam-se apenas dois instrumentos de avaliação específica da memória visual de curto prazo, com parecer favorável, destinados à avaliação do público infantojuvenil (seis a 16 anos), são eles: Figuras Complexas de Rey (Oliveira \& Rigoni, 2010), que avalia percepção e memória visual, e o Teste de Retenção visual de Benton (Sivan, 2015), que investiga a memória visual, percepção visual e praxia visuoconstrutiva.

Nesse sentido, destaca-se a relevância do Teste Pictórico de Memória (TEPIC-M), desenvolvido por Rueda e Sisto (2007), cujo objetivo é avaliar a dimensão visual da memória de curto prazo, mas não o esboço visuoespacial da memória de trabalho. O instrumento é composto por um quadro com diversas imagens, as quais devem ser observadas pelo sujeito para que, posteriormente, esse evoque e registre, por escrito, a maior quantidade de itens que consegue recuperar.

Torna-se importante ressaltar, como argumentado pelos próprios autores, que é possível distinguir tarefas de memória de curto prazo de tarefas de memória de trabalho. A primeira se refere a um sistema de armazenamento considerado passivo, envolvendo a recordação da informação visual sem que haja manipulação da mesma (Rueda \& Sisto, 2007). Os resultados oriundos do teste estabelecem uma resposta que pode ser considerada visual pelo número de objetos recordados, ou seja, o que se recorda (aspecto visual), sem que haja determinação da localização do objeto na lâmina (aspecto espacial). Convém destacar que as respostas são produzidas de forma escrita, o que demanda habilidades verbais, notadamente em termos da transformação das imagens visuais em palavras (Tormin, Cunha, \& Lopes, 2008). 
A imagem estímulo do teste consiste em 55 itens-figura com desenhos e detalhes que podem ser agrupados em três categorias: água, céu e terra. No grupo de elementos relacionados à água, é possível encontrar figuras como: surfista, jet-ski, barco à vela e peixe. Os elementos relacionados ao céu podem ser exemplificados por figuras como: sol, nuvem, gaivota e pipa. Por fim, as figuras que se referem à terra são, por exemplo: casa, fogueira, barraca e gangorra (Rueda \& Sisto, 2007).

A aplicação do teste pode ser feita de forma individual ou coletiva, em ambos é utilizado cronômetro para o cômputo do tempo total de execução do teste que é de três minutos, sendo um minuto para a exposição do quadro ao sujeito e dois minutos para registro por escrito dos itens evocados. Os estudos iniciais de validade, precisão e normatização foram realizados com sujeitos dos sexos feminino e masculino, entre 17 e 97 anos. Ressaltase que no estudo de validade, os autores submeteram inicialmente um quadro contendo 60 itens a três juízes profissionais da psicologia. Estes sugeriram a retirada de cinco itens e consideraram que os 55 restantes eram claros e de fácil acesso para a população estudada, sendo possível utilizá-los para qualquer faixa etária e nível de escolaridade (Rueda \& Sisto, 2007).

O TEPIC-M tem sido utilizado para investigação da memória visual em diferentes contextos da vida humana, tais como atividades laborais (Tormin et al., 2008) e contextos avaliativos (Rueda, 2009). Adicionalmente, foi aplicado em pesquisas que avaliam a relação da memória visual com outros construtos, notadamente a inteligência (Rueda, Cecílio-Fernandes, \& Sisto, 2008).

Diante do exposto, considerando-se a escassez de instrumentos específicos para avaliar a memória visual de curto prazo, em especial junto ao público infantojuvenil, bem como a adequabilidade dos itens e instruções do teste para todas as faixas etárias e níveis de escolaridade, objetivou-se apresentar dados normativos do Teste TEPIC-M para crianças e adolescentes. Trata-se de estudo realizado a partir de uma amostra não clínica, aleatória e estratificada por critérios de idade, sexo e tipo de escola (pública e privada) da cidade Natal (Rio Grande do Norte - RN).

\section{Método}

\section{Participantes}

Participaram deste estudo 542 estudantes, sendo 301 do sexo feminino, com idades entre seis e 16 anos, dos quais 256 eram matriculados em instituições públicas e 286 em escolas privadas do município de Natal, RN. O número total de sujeitos foi estabelecido com base no cálculo da amostra total de estudantes da cidade de Natal, com base em Levine, Berenson, e Stephan (2000). Foi encontrado o valor de 383 sujeitos, com um mínimo de 17 sujeitos por grupo, considerando-se as idades e o tipo de escola. Todavia, como se trata de amostra aleatória, optou-se por aumentar a totalidade de sujeitos em cada grupo, robustecendo assim as análises realizadas a partir dos desempenhos dos mesmos.

Os critérios de inclusão para participação nesse estudo foram: 1. Assinatura do termo de consentimento Livre e Esclarecido (TCLE) pelos pais ou responsáveis; 2. Assinatura do Termo de Assentimento Livre e Esclarecido (TALE) pelas crianças e adolescentes; 3. Relato dos professores e da coordenação das escolas sobre ausência de indicadores de alterações no desenvolvimento e/ou dificuldades escolares; 4. Inserção na rede regular de ensino. Foram excluídas as crianças e adolescentes que apresentavam dificuldades psicomotoras, auditivas e/ou visuais sem correção, que poderiam prejudicar a sua participação plena no estudo. O estudo foi aprovado pelo Comitê de Ética em Pesquisa da Universidade Federal do Rio Grande do Norte (CAEE - 50197415.9.0000.5537).

\section{Instrumentos}

Os instrumentos utilizados no presente estudo foram o TEPIC-M e um questionário sociodemográfico. Estes estão descritos a seguir.

Instrumento A. Questionário semiestruturado: composto por questões destinadas aos alunos e aos professores, investigando dados sociodemográficos (idade, sexo, tipo de escola, escolaridade) e presença/ausência de alterações neurológicas ou psiquiátricas, bem como histórico de repetência.

Instrumento B. TEPIC-M: teste desenvolvido por Rueda e Sisto (2007), cujo objetivo é a investigação da memória visual de curto prazo por meio da evocação de elementos pictóricos. O material de aplicação é constituído por um quadro contendo 55 itens distribuídos em três dimensões: terra (26 itens), céu (13 itens) e mar (16 itens). O sujeito deve observar o quadro por um tempo total de um minuto e ao longo de dois minutos registrar em uma folha de papel os itens que consegue recordar. O coeficiente de precisão do teste, aferido pelo método de duas metades de Spearman e Brown, é de 0,74 . O manual do teste traz os estudos de validade realizados, a saber, validade de constructo, interna e cruzada. Na versão original, o instrumento foi validado para o público de jovens, adultos e idosos, com faixa etária entre 17 e 97 anos. Porém, como ressaltado anteriormente, a avaliação realizada por juízes indicou que os itens que compunham o teste eram indicados para qualquer faixa etária, uma vez que foram considerados simples e claros.

\section{Procedimentos}

Inicialmente, estabeleceu-se contato com as nove escolas parceiras deste estudo, sendo cinco públicas e quatro privadas, todas da cidade de Natal (RN). Após a assinatura do Termo de Anuência pelas escolas, realizou-se reunião conjunta com professores e pais de alunos, na 
qual foi explicitado o objetivo da pesquisa e solicitada a assinatura do TCLE. Posteriormente, os objetivos foram apresentados para os alunos das turmas participantes. Os que desejaram participar assinaram o TALE e participaram da sessão de aplicação do TEPIC-M.

Os instrumentos foram aplicados nas escolas, em horário previamente acordado com todas as partes, em sala específica destinada pela coordenação, atentando-se para o nível de ruído e adequação de iluminação e projeção. As aplicações ocorreram de forma coletiva, seguindo o procedimento de Rueda e Sisto (2007), com número máximo 15 participantes por sessão, separados por faixa etária. Para os alunos de seis e sete anos, foram feitas de forma individual, com as respostas sendo registradas pelo avaliador. Tal escolha se justifica pelo fato de que nesta etapa do desenvolvimento, em especial para alunos das escolas públicas, as crianças ainda não possuem escrita automatizada, podendo a baixa velocidade do registro escrito comprometer o resultado final. O desempenho dos sujeitos foi avaliado de acordo com os critérios previstos no manual do teste, para os quais a pontuação total corresponde ao número de itens evocados corretamente.

\section{Análise de dados}

Os dados referentes aos desempenhos dos sujeitos no TEPIC-M, considerando o total de itens evocados corretamente, foram tabulados e analisados com suporte do Statistical Package for Social Sciences (SPSS), versão 20.0. Investigou-se, inicialmente, medidas de tendência central e dispersão, tais como média, mediana, moda, desvio padrão, erro padrão, percentil, escore mínimo e máximo, bem como assimetria e curtose para investigação da distribuição de frequência dos acertos e as características do achatamento da curva desta, associados ao histograma, uma vez que a amostra total possui mais de 300 sujeitos.

Quanto à análise estatística inferencial, considerando-se os resultados da assimetria e curtose que indicaram a presença de normalidade nesse perfil, procedeu-se à execução da Correlação de Pearson, para verificar medidas de validade, utilizando as variáveis idade, tipo de escola e total de acertos. O Teste $t$, para verificar diferença entre o total de acertos dos grupos, primeiro com relação ao sexo e depois ao tipo de escola. Por fim, foi realizada Análise de Variância (Anova) para verificar possíveis efeitos isolados e de interação entre as variáveis sexo, tipo de escola e nível de escolaridade sobre o desempenho dos estudantes no TEPIC-M e análise post hoc (Teste de Tukey).

\section{Resultados}

Os dados foram analisados com base nos objetivos do estudo, utilizando-se estatística descritiva e inferencial. Na Tabela 1, são apresentadas as informações acerca das medidas de tendência central e dispersão do desempenho dos participantes no TEPIC-M, considerando-se as variáveis sexo e tipo de escola, bem como a classificação de todo o grupo, doravante denominada população geral. Verifica-se que a pontuação mínima foi constante entre os diferentes grupos apresentados, sendo um mínimo de dois itens corretos referidos. A assimetria foi positiva para todos os subgrupos, porém com valores dentro da faixa considerada de simetria $(-1>$ As $<1$ ), revelando perfil de normalidade na distribuição das frequências de acertos. Por sua vez, a curtose foi negativa $(C p<0,263)$, ou seja, a distribuição tendeu a ser platicúrtica, discretamente mais achatada do que a distribuição normal.

Tabela 1

Pontuação Total no TEPIC-M conforme Variáveis do Estudo

\begin{tabular}{lccccc}
\hline & \multicolumn{5}{c}{ Amostra total } \\
\cline { 2 - 6 } & Masculino & Feminino & Escola privada & Escola pública & Geral \\
\hline$N$ & 241 & 301 & 286 & 256 & 542 \\
Média & 11,64 & 11,60 & 12,59 & 10,53 & 11,62 \\
Erro padrão & 0,28 & 0,24 & 0,23 & 0,27 & 0,18 \\
Mediana & 12 & 11 & 12 & 10 & 12 \\
Moda & 12 & 14 & 12 & 4,33 & 12 \\
Desvio padrão & 4,36 & 4,20 & 3,97 & 02 & 4,27 \\
Mínimo & 02 & 02 & 02 & 23 & 02 \\
Máximo & 24 & 23 & 24 & 24 \\
Assimetria & 0,06 & 0,28 & 0,17 & $-0,34$ & 0,18 \\
Curtose & $-0,55$ & $-0,39$ & $-0,28$ & $-0,47$ & \\
\hline
\end{tabular}

Os resultados oriundos do teste $\mathrm{t}$ para amostras independentes apontam que não houve diferença estatisticamente significativa em termos da média do total de acertos no TEPIC-M em função da variável sexo $(t=0,11 ; p=0,17)$, mas sim em função da variável tipo de escola $(t=5,77 ; p<0,001)$, a favor da escola privada. 
Buscando-se verificar possíveis efeitos de interação entre as variáveis sexo e tipo de escola sobre o desempenho da população geral no TEPIC-M, realizou-se a Anova. Evidenciou-se que não há efeito de interação entre as variáveis $(g l=1 ; F=0,70, p=0,40)$.
Posteriormente, utilizou-se a correlação de Pearson, considerando-se o total de acertos e a variável idade em função do tipo de escola. A Tabela 2 demonstrou covariação moderada do total de acertos em função da idade.

Tabela 2

Valores da Correlação entre Total de Acertos e Idade Diferenciado Tipo de Escola

\begin{tabular}{llcc}
\hline \multirow{2}{*}{ Correlação } & & Escola pública & \multicolumn{2}{c}{ Escola privada } \\
\cline { 3 - 4 } & & Idade & Idade \\
Total de acertos & $r$ & 0,34 & 0,46 \\
& $p$ & $>0,001$ & $>0,001$ \\
& $n$ & 256 & 286 \\
\hline
\end{tabular}

A Anova foi realizada a fim de verificar evidências de validades em função do desenvolvimento e mudanças a ele associadas. Ademais, a análise post hoc (Teste Tukey) buscou identificar possíveis faixas etárias formadas a partir do desempenho no teste. O resultado mostrou diferenças significativas $(F=21,022, p<0,001)$. Com a finalidade de obter a pontuação média de quais faixas etárias poderiam explicar tais diferenças, utilizou-se a
Prova de Tukey, cujos dados são apresentados na Tabela 3. Destaca-se que, conforme anteriormente aludido, devido à adaptação realizada no procedimento de aplicação do TEPIC-M para o grupo etário seis e sete anos, as análises foram feitas em separado, para esse grupo etário. Os dados revelam que não houve diferença significativa entre os resultados obtidos pelos dois grupos $(p=0,153)$.

Tabela 3

Subgrupos de Faixas Etárias Formados pela Prova de Tukey para o TEPIC-M

\begin{tabular}{ccccc}
\hline \multirow{2}{*}{ Faixas etárias } & $n$ & \multicolumn{3}{c}{ Subgrupos para alfa=0,05 } \\
\cline { 3 - 4 } & & 8,33 & 2 \\
$10-9$ & 90 & & 10,66 \\
11 & 106 & & & 13,78 \\
$P$ & 246 & 1,00 & 1,00 & 1,00 \\
\hline
\end{tabular}

Baseando-se na categorização proposta e considerando-se as variáveis idade e tipo de escola, realizou-se análises descritivas para investigar medidas de tendência central e dispersão, tais como média, mediana, moda, desvio padrão, erro padrão, percentil, escore mínimo e máximo, conforme mostram a Tabela 4, para os diferentes grupos etários e por tipo de escola. Destaca-se que os níveis de percentis, bem como as classificações, foram baseados na proposição do estudo inicial de Rueda e Sisto (2007).

Tabela 4

Estatísticas Descritivas e Percentis para Pontuação no TEPIC-M

\begin{tabular}{lcccccccc}
\hline & \multicolumn{4}{c}{ Escola pública } & \multicolumn{4}{c}{ Escola particular } \\
\cline { 2 - 9 } & $6-7$ & $8-9$ & $10-11$ & $12-16$ & $6-7$ & $8-9$ & $10-11$ & $12-16$ \\
\hline$N$ & 56 & 78 & 52 & 103 & 44 & 75 & 54 & 143 \\
Média & 10,55 & 7,95 & 8,25 & 12,97 & 9,95 & 10,29 & 12,98 & 14,36 \\
Erro padrão & 0,57 & 0,34 & 0,43 & 0,38 & 0,48 & 0,37 & 0,48 & 0,29 \\
Mediana & 10 & 7 & 8 & 13 & 9,5 & 10 & 12 & 14 \\
Moda & 10 & 6 & 6 & 14 & 9 & 8 & 11 & 14 \\
Desvio padrão & 4,28 & 3,05 & 3,13 & 3,95 & 3,24 & 3,27 & 3,57 & 3,56 \\
\hline
\end{tabular}


Tabela 4 (continuação)

Estatísticas Descritivas e Percentis para Pontuação no TEPIC-M

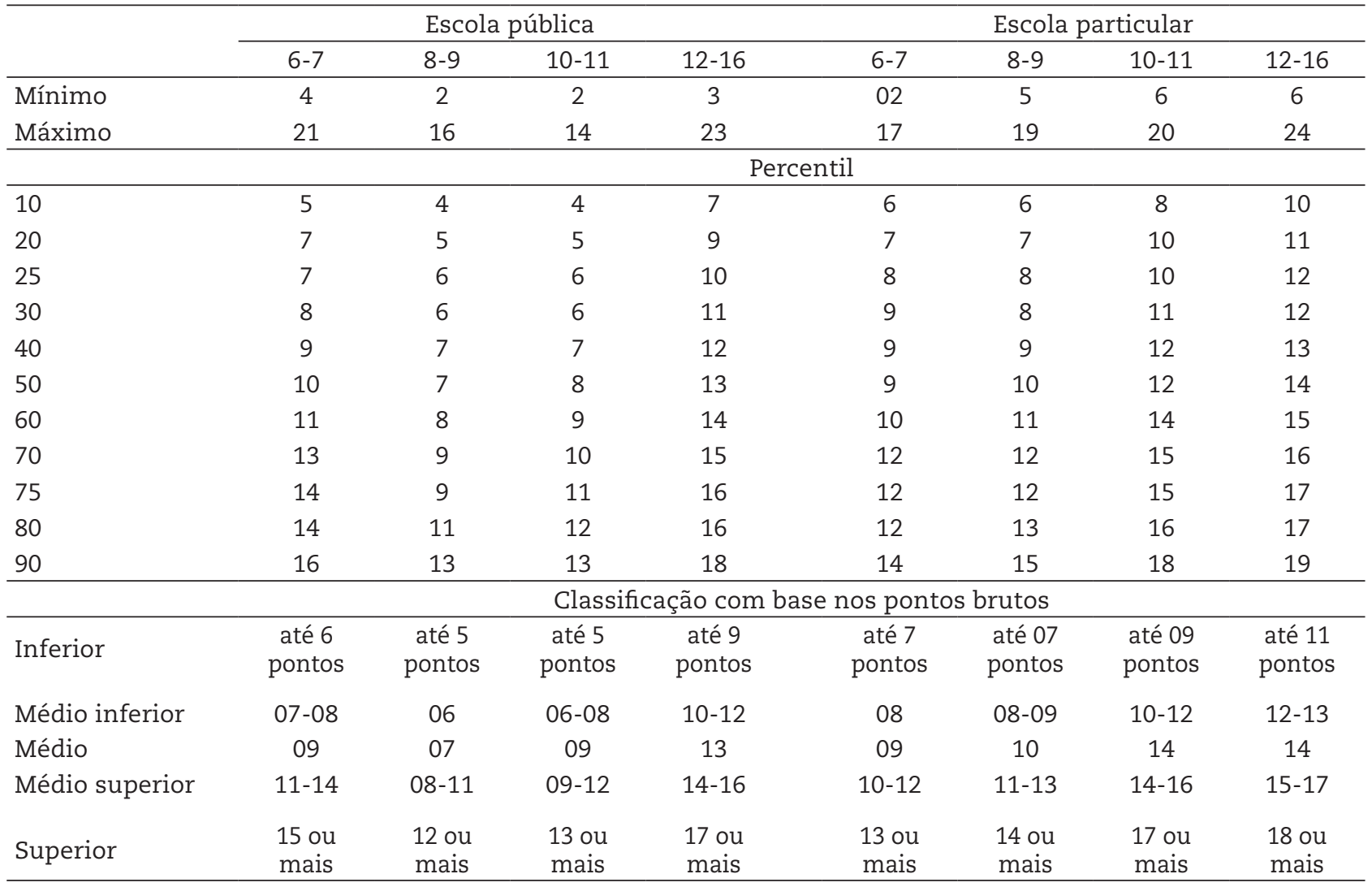

\section{Discussão}

O presente estudo apresenta dados normativos de desempenho de crianças e adolescentes em um instrumento de avaliação da memória visual de curto prazo (TEPIC-M). A escassez de instrumentos de fácil aplicação, destinados ao público infantojuvenil e que avalie o sistema de memória em tela, aponta para a sua relevância.

Participaram do estudo crianças e adolescentes de 6 a 16 anos, estudantes de instituições de ensino públicas e privadas da cidade de Natal-RN. Os dados referentes aos desempenhos dos sujeitos foram agrupados conforme a idade, o sexo e o tipo de escola (pública ou privada).

No que se refere à variável idade, é interessante destacar que nos estudos iniciais de normatização e validação do TEPIC-M, realizados com sujeitos de 17 a 97 anos, os autores encontraram uma correlação significativa e negativa entre idade e desempenho, demonstrando um declínio na quantidade de acertos com o acréscimo da idade. Esses dados corroboram achados da literatura que caracterizam o percurso de desenvolvimento da memória visual, assim como de outras funções cognitivas, com a forma de $\mathrm{U}$ invertido, ou seja, crescente durante as fases da infância, chegando ao seu ápice no início da idade adulta e declinando com o avançar da idade (Carson, Vanderhorst, \& Koenig, 2015; Montefinese, Sulpizio, Galati, \& Committeri,
2015). Todavia, apesar deste estudo ter encontrado correlação significativa e positiva entre idade e desempenho, foi identificado platô no início da adolescência, ou seja, não houve diferença estatística significativa entre os desempenhos dos grupos de sujeitos entre os 12 e 16 anos. Este é um dado relevante que merece discussão.

Estudos que investigaram a memória de trabalho, em especial o esboço visuoespacial, demonstram que esse sistema de memória tem o seu desenvolvimento prolongado ao longo de toda a adolescência, não atingindo desempenho similar ao de adultos antes dos 16 anos (Isbell, Fukuda, Neville, \& Vogel, 2015; Swanson, 2017). Entretanto, os dados aqui obtidos que, num primeiro momento, parecem ir de encontro ao posicionamento vigente. $\mathrm{Na}$ verdade, podem ser interpretados como reforço à teoria que defende a relativa independência entre a memória de curto prazo e a memória de trabalho.

Corroborando a tese supracitada, o estudo realizado por Kuhn (2016) explorou a contribuição de tarefas de armazenamento e processamento da memória de trabalho, de atenção e de memória de curto prazo para as inteligências fluida e cristalizada de 275 estudantes de 8 a 13 anos. Os resultados sugerem a existência de dois fatores estruturais da memória, sendo o primeiro fator composto pelo conjunto de tarefas que avaliaram a memória de trabalho e a atenção, e o segundo fator a memória de curto prazo. 
A memória de trabalho despontou como forte preditor das inteligências fluida e cristalizada, tanto quando modelada separadamente, quanto quando modelada como fator residual para controle da variância da memória de curto prazo. Adicionalmente, a memória de curto prazo interagiu com a variável idade e não teve relação com a inteligência fluida em crianças com mais de 11 anos. Por sua vez, o efeito da memória de trabalho não foi moderado pela idade. $\mathrm{O}$ estudo conclui que a memória de curto prazo e a memória de trabalho são sistemas distintos, mas altamente relacionados (Kuhn, 2016). Sendo assim, os dados deste estudo e da literatura apresentam evidências que as relações entre memória de trabalho visuoespacial e memória de curto prazo visual podem ser moderadas pela idade e estão sujeitas a modificações ao longo do tempo.

Em relação à variável tipo de escola, convém problematizar acerca da complexidade a ela subjacente. Isso porque não se trata apenas da qualidade do processo ensino-aprendizagem, mas de outras variáveis socioeconômicas que estão diretamente atreladas a ela, em especial na Região Nordeste do país, local de realização deste estudo. Em artigo de revisão sistemática, Ursache e Noble (2016) destacaram o quanto diferentes variáveis associadas à pobreza e à baixa estimulação impactam significativamente o desenvolvimento de funções cognitivas, dentre elas a memória, tanto no que se refere à aquisição, quanto ao declínio.

Em estudo brasileiro, realizado com 419 estudantes com idades entre seis e 12 anos, foi investigado o efeito do nível socioeconômico sobre o desempenho em tarefas de inteligência verbal, linguagem, memória e funções executivas. Os resultados sugerem que a variável nível socioeconômico contribui para o desempenho em todas as medidas, incluindo a memória, em todas as faixas etárias consideradas (Piccolo, Arteche, Fonseca, GrassiOliveira, \& Salles, 2016).

O fato de o TEPIC-M apontar diferenças e mudanças de desempenho em função das variáveis idade e tipo de escola sugerem evidências de validade, especialmente nos anos iniciais do desenvolvimento. No entanto, convém destacar que se trata de estudo realizado em Região específica do país, caracterizada pelo baixo Índice de Desenvolvimento Humano (IDH) e pela alta desigualdade de ofertas de saúde educação para a população (Rodrigues, 2018), o que dificulta a generalização dos achados para a realidade brasileira, evidenciando a necessidade de ampliação dos estudos normativos do TEPIC-M para outras regiões do país. Tal reflexão aponta para a complexidade do desenvolvimento humano e a necessidade de considerar esse fenômeno a partir da realidade histórico-cultural na qual a criança está imersa. Do contrário, corre-se o risco de realização de análises generalistas que contribuem para segregação e para descrédito da qualidade do ensino público brasileiro.

\section{Considerações Finais}

Diante da escassez de pesquisas e instrumentos que avaliem a memória na infância e adolescência, assim como se reconhecendo que diferentes sistemas e subsistemas mnêmicos se modificam ao longo do desenvolvimento, torna-se necessário o aprimoramento de instrumentos que possibilitem a avaliação da função cognitiva em questão (Santos, Andrade, \& Bueno, 2015).

Os dados apresentados no presente estudo objetivam colaborar para a minimização da realidade supracitada, ofertando evidências de validade de critério e parâmetros normativos do teste TEPIC-M, desenvolvido para a avaliação da memória visual de curto prazo. Inicialmente, o instrumento foi validado e normatizado para jovens, adultos e idosos de 17 a 97 anos. Os dados ora disponibilizados contemplam crianças e adolescentes de seis a 16 anos.

Os resultados encontrados sugerem que o TEPIC-M possui sensibilidade diante das variações de idade, ou seja, para as mudanças que ocorrem nos sistemas de memória subjacentes ao desenvolvimento, em especial, nas fases iniciais. Adicionalmente, a ausência de dificuldades na execução da tarefa proposta corrobora o posicionamento dos juízes que, na etapa de validação do instrumento, ressaltaram que os estímulos que compunham o quadro de referência do teste eram adequados para todas as idades, ainda que os estudos de validação iniciais tenham sido circunscritos à faixa etária dos 17 a 97 anos.

É importante destacar que o estudo possui limitações em sua execução. Enfatiza-se, inicialmente, que a amostra de estudantes que integrou a pesquisa foi constituída por crianças e adolescentes de uma capital da Região Nordeste do Brasil. Como discutido anteriormente, esta é caracterizada pelos piores índices de desenvolvimento humano do Brasil, o que pode impactar diretamente o desenvolvimento de várias funções cognitivas, dentre elas a memória. Nesse sentido, os dados ora apresentados são limitados e seu uso não deve ser estendido diretamente para as demais as regiões do país.

Contudo, ressalta-se a importância de estudos normativos com dados específicos para o Nordeste do Brasil. De modo geral, as normatizações de instrumentos no país são realizadas nas Regiões Sudeste e Sul. E, uma vez que a limitação supracitada e o cuidado no uso dos dados ora apresentados devem ser considerados para as demais regiões do país, o mesmo zelo deve ser tomado por pesquisadores e clínicos de outras regiões brasileiras, distintas destas, que fizerem uso de testes cuja normatização não contemplou sujeitos de suas regiões.

Outra limitação importante se refere à ausência da avaliação dos níveis de inteligência e de habilidade escrita da amostra, visto que os estudos apontam para relação positiva e significativa entre a inteligência fluida e 
a memória visual de curto prazo, em especial nos anos iniciais (Isbell et al., 2015; Kuhn, 2016; Swanson, 2017). Estes sugerem que a memória possui importante papel na verificação de dificuldades relacionadas à aprendizagem, além de sensibilidade na diferenciação do funcionamento intelectivo e na averiguação dos prejuízos decorrentes de quadros neurológicos (Oliveira \& Ciasca, 2015; Pereira, Araújo, Ciasca, \& Rodrigues, 2015). Nesse sentido, estudantes que integraram a amostra do estudo, com valores de quociente de inteligência (QI) fora da variação normal, para mais ou menos, podem ter contribuído para o aumento ou rebaixamento das médias do grupo dos quais fizeram parte. Por outro lado, dificuldades de escrita podem ter comprometido a produção final em termos de palavras registradas.
A partir das reflexões avançadas, aponta-se a importância da realização de estudos normativos do TEPIC-M com crianças e adolescentes de outras regiões do Brasil, para - em um primeiro momento analisar se há diferença entre o total de acertos, bem como nos diferentes elementos evocados. Para além de estudos normativos e transculturais, também é possível elaborar pesquisas utilizando os dados aqui apresentados com grupos clínicos específicos, como crianças e adolescentes com deficiência intelectual, transtornos de aprendizagem, transtornos do neurodesenvolvimento e altas habilidades/superdotação. Tais iniciativas serão decisivas para robustecer a prática clínica e de pesquisa em diferentes domínios da avaliação e intervenção psicológica.

\section{Referências}

Abreu, N., Lima, C., Siquara, G., Wyzykowski, A., \& Fonseca. M. (2018). Memória. In L. Malloy-Diniz, D. Fuentes, P. Mattos, \& N. Abreu. (Eds.). Avaliação Neuropsicológica (pp. 73-82). Porto Alegre: Artmed.

Atkinson, R. C., \& Shiffrin, R. M. (1968). Human memory: A proposed system and its control procesrses. In K. W. Spence \& J. T. Spence (Eds.), The psychology of learning and motivation: II (pp. 89-195). Oxford, UK: Academic Press. doi: 10.1016/S0079-7421(08)60422-3

Baddeley, A., \& Hitch, G. (1974). Working memory. In G. A. Bower (Ed.), Recent advances in learning and motivation (pp. 47-89). Academic Press: New York. doi: 10.1016/s0079-7421(08)60452-1

Baddeley, A. D. (2000). The episodic buffer: A new component of working memory? Trends in Cognitive Sciences, 4(11), 417-423. doi: 10.1016/S1364-6613(00)01538-2

Baddeley, A. (2012). Working memory: Theories, models, and controversies. Annual Review of Psychology, 63(1), 1-29. doi: 10.1146/annurevpsych-120710-100422

Brockmole, J. R., \& Logie, R. H. (2013). Age-related change in visual working memory: A study of 55,753 participants aged 8-75. Frontiers in Psychology, 4(12), 1-5. doi: 10.3389/fpsyg.2013.00012

Carson, V. B., Vanderhorst, K. J., \& Koenig, H. G. (2015). Care Giving for Alzheimer's Disease: A compassionate guide for clinicians and loved ones. New York: Springer Science.

Eysenck, M. (2017). Manual de Psicologia Cognitiva. Porto Alegre: Artmed.

Garcia, R. B., Mammarella, I. C., Pancera, A., Galera, C., \& Cornoldi, C. (2015). Deficits in visual short-term memory binding in children at risk of non-verbal learning disabilities. Research in Developmental Disabilities, 45-46, 365-372. doi: 10.1016/j.ridd.2015.07.035

Isbell, E., Fukuda, K., Neville, H. J., \& Vogel, E. K. (2015). Visual working memory continues to develop through adolescence. Frontiers in Psychology, 6(696), 1-10. doi: 10.3389/fpsyg.2015.00696

Jonides, J., Lewis, R., Nee, D., Lusting, C., Berman, M., \& Moore, K. (2008). The mind and brain of sjort-term memory. Annual Review of Psychology, 59, 193-224. doi: 10.1146/annurev.psych.59.103006.093615.

Kuhn, J. (2016). Controlled attention and storage: An investigation of the relationship between working memory, short-term memory, scope of attention, and intelligence in children. Learning and Indivudual Differences, 52, 167-177. doi: 10.1016/j.lindif.2015.04.009

Levine, D. M., Berenson, M. L., \& Stephan, D. (2000). Estatística: Teoria e aplicações, usando Microsoft Excel em Português. Rio de Janeiro, Brasil: LTC.

Montefinese, M., Sulpizio, V., Galati, G., \& Committeri, G. (2015). Age-related effects on spatial memory across viewpoint changes relative to different reference frames. Psychological Research, 79(4), 687-697. doi: 10.1007/s00426-014-0598-9

Montoya, M., Susperreguy, M., Dinarte, L., Morrison, F., Martin, E., Barahona, C., ... Forster, C. (2019). Executive function in Chilean preschool children: Do short-term memory, working memory, and response inhibition contribute differentially to early academic skills? Early Childhood Research Quartely, 46, 187-200. doi:10.1016/j.ecresq.2018.02.009

Oliveira, J. A., \& Ciasca, S. M. (2015). Avaliação da memória em crianças e adolescentes com histórico de acidente vascular cerebral e crianças com queixas de dificuldades escolares. Revista Psicopedagogia, 32(98), 128-135. Recuperado de http://pepsic.bvsalud.org/scielo. php? script $=$ sci_arttext\&pid $=$ S0103-84862015000200003\&lng $=$ pt\&tlng $=$ pt.

Oliveira, M. S. \& Rigoni, M. S. (2010). Figuras Complexas de Rey: Teste de Cópia e de Reprodução de Memória de Figuras Geométricas Complexas. São Paulo: Casa do Psicólogo.

Pereira, A. M., Araújo, C. R., Ciasca, S. M., \& Rodrigues, S. (2015). Avaliação da memória em crianças e adolescentes com capacidade intelectual limítrofe e deficiência intelectual leve. Revista Psicopedagogia, 32(99), 302-313. Recuperado de http://pepsic.bvsalud.org/ scielo.php? script $=$ sci_arttext\&pid $=$ S0103-84862015000300004\&lng $=$ pt\&tlng $=$ pt.

Piccolo, L., Arteche, A., Fonseca, R., Grassi-Oliveira, R., \& Salles, J. (2016). Influence of family socioeconomic status on IQ, language, memory and executive functions of Brazilian children. Psicologia: Reflexão e Crítica, 29(23), 1-10. doi: 10.1186/s41155-016-0016-x

Roberts, K. L., Strait, J. A. E., \& Decker, S. L. (2018). Developmental trajectories of verbal, static visual-spatial, and dynamic visual-spatial working memory. Contemporary School Psychology, 22(4), 458-467. doi: 10.1007/s40688-018-0176-z 
Rodrigues, W. (2018). Capital social e desenvolvimento regional no Brasil. Revista Brasileira de Gestão e Desenvolvimento Regional, 14(1), 43-60. Recuperado de https://www.rbgdr.net/revista/index.php/rbgdr/article/view/3466

Rueda, F. (2009). Atenção concentrada e memória: Evidências de validade entre instrumentos no contexto da psicologia do trânsito. Psicologia Teoria e Pesquisa, 11(2), 182-195. Recuperado de http://pepsic.bvsalud.org/scielo.php?script=sci_arttext\&pid=S1516$36872009000200013 \& \operatorname{lng}=\mathrm{pt} \& \mathrm{tlng}=\mathrm{pt}$.

Rueda, F., Cecilio-Fernandes, D., \& Sisto, F. F. (2008). Memória pictórica e inteligência: Duas evidências de validade. Estudos e Pesquisas em Psicologia, 8(3), 774-788. Recuperado de http://pepsic.bvsalud.org/scielo.php?script=sci_arttext\&pid=S180842812008000300015\&lng $=$ pt\&tlng $=$.

Rueda, F., \& Sisto, F. (2007). Teste Pictórico de Memória - (TEPIC-M): Manual. São Paulo, Brasil: Vetor.

Santos, F. H., Andrade, V. M., \& Bueno, O. F. (2004). Neuropsicologia hoje. São Paulo, Brasil: Artes Médicas.

Sivan, A. B. (2015). Teste de Retenção Visual de Benton-BVRT. (J. F. de Salles, D. R. Bandeira, C. M. Trentini, J. D. Segabinazi, \& C. S. Hutz, trad.). São Paulo, Brasil: Vetor Editora.

Swanson, H. (2017). Verbal and visual-spatial working memory: What develops over a life span? Developmental Psychology, 51(5), 971-995. doi: $10.1037 / \operatorname{dev} 0000291$

Tormin, M. C., Cunha, C. A., \& Lopes, R. F. (2008). Adaptação do Teste Pictórico de Memória para avaliação da memória de trabalho em musicistas. Revista de Psicologia da Vetor Editora, 9(1), 89-98. Recuperado de http://pepsic.bvsalud.org/scielo.php?script=sci_ arttext\&pid=S1676-73142008000100011\&lng = pt\&tlng=pt.

Ursache, A., \& Noble, K. G. (2016). Neurocognitive development in socioeconomic context: Multiple mechanisms and implications for measuring socioeconomic status. Psychophysiology, 53(1), 71-82. doi: 10.1111/psyp.12547

\section{Sobre os autores}

Luana Metta é psicóloga (UFRN), mestra em Psicologia pela Universidade Federal do Rio Grande do Norte e, atualmente, doutoranda pelo Programa de Pós-Graduação em Psicologia da Universidade Federal do Rio Grande do Norte (UFRN).

Rodrigo da Silva Maia é psicólogo (UFRN), doutor em Psicologia pelo Programa de Pós-Graduação em Psicologia da Universidade Federal do Rio Grande do Norte e, atualmente, é professor adjunto do curso de Psicologia da Universidade Federal do Ceará (UFC), Campus Sobral.

Daniele Caroline Leôncio é psicóloga (UFRN), mestra em Psicologia pela Universidade Federal do Rio Grande do Norte e, atualmente, doutoranda pelo Programa de Pós-Graduação em Psicologia da Universidade Federal do Rio Grande do Norte (UFRN).

Laura Aragão é psicóloga (UFRN), mestra em Psicologia pela Universidade Federal do Rio Grande do Norte e, atualmente, doutoranda pelo Programa de Pós-Graduação em Psicologia da Universidade Federal do Rio Grande do Norte (UFRN).

Hedyanne Guerra Pereira é psicóloga (UFRN), mestra em Psicologia pela Universidade Federal do Rio Grande do Norte e, atualmente, é doutoranda pelo Programa de Pós-Graduação em Psicologia da Universidade Federal do Rio Grande do Norte (UFRN).

Eulália Maria Chaves Maia é psicóloga (Instituto Paraibanos de Educação), doutora em Psicologia Clínica (USP/SP). Atualmente, é professora titular e bolsista de produtividade (CNPq), vinculada ao Programa de Pós-Graduação em Psicologia da Universidade Federal do Rio Grande do Norte (UFRN).

Izabel Hazin é psicóloga (PUC-SP), doutora em Psicologia Cognitiva pela Universidade Federal de Pernambuco e pós-doutorada pela Universitè René Descartes - Paris V. Atualmente, é professora associada 2 do Departamento de Psicologia e bolsista de produtividade (CNPq), vinculada ao Programa de Pós-Graduação em Psicologia da UFRN. 